\title{
Logarithmic density and logarithmic statistical convergence
}

\author{
Mohammed A Alghamdi ${ }^{1}$, Momammad Mursaleen ${ }^{2 *}$ and Abdullah Alotaibi ${ }^{1}$
}

"Correspondence:

mursaleenm@gmail.com

${ }^{2}$ Department of Mathematics, Aligarh Muslim University, Aligarh, 202002, India

Full list of author information is available at the end of the article

\begin{abstract}
In this paper we use the idea of logarithmic density to define the concept of logarithmic statistical convergence. We find the relations of logarithmic statistical convergence with statistical convergence, statistical summability $(H, 1)$ introduced by Móricz (Analysis 24:127-145, 2004) and $\left[H_{1} 1\right]_{q}$-summability. We also give subsequence characterization of statistical summability $(H, 1)$.
\end{abstract}

MSC: 40A05; 40A30

Keywords: asymptotic density; logarithmic density; statistical convergence; statistical summability $(H, 1)$; logarithmic statistical convergence

\section{Introduction and preliminaries}

The concept of statistical summability $(H, 1)$, which is a generalization of statistical convergence due to Fast [1], has recently been introduced by Móricz [2]. In this paper we use the idea of logarithmic density to define the concept of logarithmic statistical convergence. We find its relation with statistical convergence and statistical summability $(H, 1)$. We further define $[H, 1]_{q}$-summability and establish some inclusion relations.

Definition 1.1 Let $\mathbb{N}$ be the set of all natural numbers and let $\chi_{E}$ denote the characteristic function of $E \subseteq \mathbb{N}$. Put $d_{n}(E)=\frac{1}{n} \sum_{k=1}^{n} \chi_{E}(k)$ and $\delta_{n}(E)=\frac{1}{l_{n}} \sum_{k=1}^{n} \frac{\chi_{E}(k)}{k}$ for $n \in \mathbb{N}$, where $l_{n}=\sum_{k=1}^{n} 1 / k(n=1,2,3, \ldots)$. The numbers $\underline{d}(E)=\liminf _{n \rightarrow \infty} d_{n}(E)$ and $\bar{d}(E)=$ $\lim \sup _{n \rightarrow \infty} d_{n}(E)$ are called the lower and upper asymptotic density of $E$, respectively. Similarly, the numbers $\underline{\delta}(E)=\liminf _{n \rightarrow \infty} \delta_{n}(E)$ and $\bar{\delta}(E)=\limsup _{n \rightarrow \infty} \delta_{n}(E)$ are called the lower and upper logarithmic density of $E$, respectively. If $\underline{d}(E)=\bar{d}(E)=d(E)$, then $d(E)$ is called the asymptotic density of $E\left(\underline{\delta}(E)=\bar{\delta}(E)=\delta_{\ln }(E)\right.$ is called the logarithmic density of $E$, respectively).

Note that for $k=1, l_{n}=\sum_{k=1}^{n} 1 / k=n$ and hence $\delta_{\ln }(E)$ reduces to $d(E)$.

Now recall the concept of statistical convergence of real sequences (see Fast [1] and Fridy [3]).

Definition 1.2 A sequence $x=\left(x_{k}\right)$ is said to be statistically convergent to $L$ if for every $\epsilon>0, d\left(\left\{k:\left|x_{k}-L\right| \geq \epsilon\right\}\right)=0$. That is,

$$
\lim _{n} \frac{1}{n}\left|\left\{k \leq n:\left|x_{k}-L\right| \geq \epsilon\right\}\right|=0
$$

(0) 2013 Alghamdi et al.; licensee Springer. This is an Open Access article distributed under the terms of the Creative Commons Attribution License (http://creativecommons.org/licenses/by/2.0), which permits unrestricted use, distribution, and reproduction in any medium, provided the original work is properly cited. 
Several extensions, variants and generalizations of this notion have been investigated by various authors, namely [2, 4-16].

\section{Logarithmic statistical convergence}

In this section we define the logarithmic statistical convergence and $[H, 1]_{q}$-summability and establish some inclusion relations.

Definition 2.1 A sequence $x=\left(x_{k}\right)$ is said to be logarithmic statistically convergent to $L$ if for every $\epsilon>0$, the set $\left\{k:\left|x_{k}-L\right| \geq \epsilon\right\}$ has logarithmic density zero. That is,

$$
\lim _{n} \frac{1}{l_{n}}\left|\left\{k \leq n: \frac{1}{k}\left|x_{k}-L\right| \geq \epsilon\right\}\right|=0 .
$$

In this case we write $s t_{\ln }-\lim x=L$ and we denote the set of all logarithmic statistically convergent sequences by $s t_{\mathrm{ln}}$.

Remark 2.1 One can say that logarithmic statistical convergence is a special case of weighted statistical convergence [15] if $p_{k}=\frac{1}{k}$. But this is not exactly true, since for $p_{k}=\frac{1}{k}, P_{n}=\sum_{k=1}^{n} p_{k}=\sum_{k=1}^{n} 1 / k \approx \log n(n=1,2,3, \ldots)$, and consequently, the definition of weighted statistical convergence gives that $\lim _{n} \frac{1}{l_{n}}\left|\left\{k \leq l_{n} \approx \log n: \frac{1}{k}\left|x_{k}-L\right| \geq \epsilon\right\}\right|=0$. So, one can see the difference between this and (2.1), i.e., in (2.1) the enclosed set has bigger cardinality.

Definition 2.2 Let $\tau_{n}:=l_{n}^{-1} \sum_{k=1}^{n} x_{k} / k$, where $l_{n}=\sum_{k=1}^{n} 1 / k \approx \log n(n=1,2,3, \ldots)$. We say that $x=\left(x_{k}\right)$ is $(H, 1)$-summable to $L$ if the sequence $\tau=\left(\tau_{n}\right)$ converges to $L$, i.e., $(H, 1)-\lim x=L$.

If $k=1$ then $l_{n}=\sum_{k=1}^{n} 1 / k=n$, and $(H, 1)$-summability is reduced to $(C, 1)$-summability.

Definition 2.3 A sequence $x=\left(x_{k}\right)$ is said to be $[H, 1]_{q}$-summable $(0<q<\infty)$ to the limit $L$ if $\lim _{n} \frac{1}{l_{n}} \sum_{k=1}^{n} \frac{1}{k}\left|x_{k}-L\right|^{q}=0$, and we write it as $x_{k} \rightarrow L[H, 1]_{q}$. In this case $L$ is called the $[H, 1]_{q}$-limit of $x$.

Let $q=1$. If $k=1$ then $l_{n}=\sum_{k=1}^{n} 1 / k=n,[H, 1]_{q}$-summability is reduced to strong $(C, 1)$ summability. Also, $[H, 1]_{q}$-summability is a special case of $\left[\bar{N}, p_{n}\right]_{q}$-summability (cf. [15]) for $p_{k}=\frac{1}{k}$.

Recently, Móricz [2] has defined the concept of statistical summability $(H, 1)$ as follows.

Definition 2.4 A sequence $x=\left(x_{k}\right)$ is said to be statistically summable $(H, 1)$ to $L$ if the sequence $\tau=\left(\tau_{n}\right)$ is statistically convergent to $L$, i.e., st- $\lim \tau=L=H(s t)-\lim x$. We denote by $H(s t)$ the set of all sequences which are statistically summable $(H, 1)$ and we call such sequences statistically $(H, 1)$-summable sequences.

Remark 2.2 If $x=\left(x_{k}\right)$ is bounded, then $s t-\lim _{k \rightarrow \infty} x_{k}=L$ implies $(C, 1)-\lim _{k \rightarrow \infty} x_{k}=L$ (see [17]). The converse is obviously not true, e.g., $x=(1,0,1,0, \ldots)$ is $(C, 1)$-summable to $\frac{1}{2}$ but not statistically convergent. However, for bounded sequences, statistical convergence to some number is equivalent to strong Cesàro summability to the same number. But for logarithmic statistical convergence the situation is different (see [8]). 
Theorem 2.1 Statistical convergence implies logarithmic statistical convergence but converse need not be true.

Proof It is well known that for each $E \subseteq \mathbb{N}, \underline{d}(E) \leq \underline{\delta}(E) \leq \bar{\delta}(E) \leq \bar{d}(E)$ (see [18], pp.70-75, pp.95-96). Hence if $d(E)$ exists, then also $\delta_{\ln }(E)$ exists and $d(E)=\delta_{\ln }(E)$. Hence statistical convergence implies logarithmic statistical convergence.

Take $E_{k}=\left\{k^{k^{2}}+1, k^{k^{2}}+2, \ldots, k^{k^{2}+1}\right\}(k \in \mathbb{N})$ and $E=\bigcup_{k=2}^{\infty} E_{k}$. If $E(n)=d_{n}(E)$ for $n \in \mathbb{N}$, then

$$
\bar{d}(E) \geq \lim \sup _{k \rightarrow \infty} \frac{E\left(k^{k^{2}+1}\right)}{k^{k^{2}+1}} \geq \lim \sup _{k \rightarrow \infty} \frac{k^{k^{2}+1}-k^{k^{2}}}{k^{k^{2}+1}}=1 .
$$

Hence $\bar{d}(E)=1$.

Since $\sum_{j \in E_{n}} \frac{1}{j}=\ln k+O\left(\frac{1}{k^{k^{2}}}\right)(k \in \mathbb{N}, k \geq 2)$, we get

$$
\bar{\delta}(E) \leq \lim _{n \rightarrow \infty} \frac{\sum_{k=1}^{n} \ln k+O(1)}{\sum_{j=1}^{n^{n^{2}+1}} \frac{1}{j}} \leq \lim \frac{n \ln n+O(1)}{\left(n^{2}+1\right) \ln n+O(1)}=0 .
$$

Hence $\delta_{\ln }(E)=0$ and consequently $\underline{d}(E)=0$, i.e., $d(E)$ does not exist. Define the sequence $x=\left(x_{k}\right)$ by

$$
x_{k}= \begin{cases}1 & \text { if } k \in E \\ 0 & \text { if } k \in \mathbb{N} \backslash E .\end{cases}
$$

Since $\delta_{\ln }(E)=0$, we have $s t_{\ln }-\lim _{n \rightarrow \infty} x_{n}=0$. But $(C, 1)$ - $\lim _{n \rightarrow \infty} x_{n}$ does not exist because $\frac{1}{n} \sum_{m=1}^{n} x_{m}=\frac{E(n)}{n}(n \in \mathbb{N})$ and hence $s t-\lim _{n \rightarrow \infty} x_{n}$ does not exist.

This completes the proof.

\section{Main results}

In the following theorem we establish the relation between logarithmic statistical convergence and Móricz's statistical summability $(H, 1)$.

Theorem 3.1 If a sequence $x=\left(x_{k}\right)$ is bounded and logarithmic statistically convergent to $L$ then it is statistically summable $(H, 1)$ to $L$, but not conversely.

Proof Let $x=\left(x_{k}\right)$ be bounded and logarithmic statistically convergent to $L$. Write $K_{\epsilon}:=$ $\left\{k \in \mathbb{N}: \frac{1}{k}\left|x_{k}-L\right| \geq \epsilon\right\}$. Then

$$
\begin{aligned}
\left|\tau_{n}-L\right| & =\left|l_{n}^{-1} \sum_{k=1}^{n} x_{k} / k-L\right|=\left|l_{n}^{-1} \sum_{k=1}^{n} \frac{1}{k}\left(x_{k}-L\right)\right| \leq l_{n}^{-1} \sum_{k=1}^{n} \frac{1}{k}\left|x_{k}-L\right| \\
& \leq l_{n}^{-1} \sum_{k \in K_{\epsilon}}\left|x_{k}-L\right| \leq l_{n}^{-1}\left(\sup _{k}\left|x_{k}-L\right|\right) K_{\epsilon} \rightarrow 0
\end{aligned}
$$

as $n \rightarrow \infty$, which implies that $\tau_{n} \rightarrow L$ as $n \rightarrow \infty$. That is, $x$ is $(H, 1)$-summable to $L$ and hence statistically summable $(H, 1)$ to $L$. 
For converse, we consider the special case when $k=1$, then $l_{n}=n$ as above. Consider the sequence $x=\left(x_{k}\right)$ defined by

$$
x_{k}= \begin{cases}1 & \text { if } k \text { is odd } \\ 0 & \text { if } k \text { is even }\end{cases}
$$

Of course this sequence is not logarithmic statistically convergent. On the other hand, $x$ is $(H, 1)$-summable to 1 and hence statistically summable $(H, 1)$ to 1 .

This completes the proof of the theorem.

Remark 3.1 The above theorem is analogous to Theorem 2.1 of [15] but this holds for any bounded sequence.

In the next result we establish the inclusion relation between logarithmic statistical convergence and $[H, 1]_{q}$-summability.

Theorem 3.2 (a) If $0<q<\infty$ and a sequence $x=\left(x_{k}\right)$ is $[H, 1]_{q}$-summable to the limit $L$, then it is logarithmic statistically convergent to $L$.

(b) If $\left(x_{k}\right)$ is bounded and logarithmic statistically convergent to $L$, then $x_{k} \rightarrow L[H, 1]_{q}$.

Proof (a) If $0<q<\infty$ and $x_{k} \rightarrow L[H, 1]_{q}$, then

$$
\begin{aligned}
0 & \leftarrow l_{n}^{-1} \sum_{k=1}^{n} \frac{1}{k}\left|x_{k}-L\right|^{q} \geq l_{n}^{-1} \sum_{\substack{k=1 \\
\left|x_{k}\right| k-L \mid \geq \epsilon}}^{n} \frac{1}{k}\left|x_{k}-L\right|^{q} \\
& \geq \frac{\epsilon^{q}}{l_{n}}\left|K_{\epsilon}\right|
\end{aligned}
$$

as $n \rightarrow \infty$. That is, $\lim _{n \rightarrow \infty} \frac{1}{l_{n}}\left|K_{\epsilon}\right|=0$ and so $\delta_{\ln }\left(K_{\epsilon}\right)=0$, where $K_{\epsilon}:=\left\{k \leq n: \frac{1}{k}\left|x_{k}-L\right| \geq \epsilon\right\}$. Hence $x=\left(x_{k}\right)$ is logarithmic statistically convergent to $L$.

(b) Suppose that $x=\left(x_{k}\right)$ is bounded and logarithmic statistically convergent to $L$. Then, for $\epsilon>0$, we have $\delta_{\ln }\left(K_{\epsilon}\right)=0$. Since $x \in l_{\infty}$, there exists $M>0$ such that $\left|x_{k}-L\right| \leq M$ $(k=1,2, \ldots)$. We have

$$
l_{n}^{-1} \sum_{k=1}^{n} \frac{1}{k}\left|x_{k}-L\right|^{q}=\frac{1}{l_{n}} \sum_{\substack{k=1 \\ k \notin K_{\epsilon}}}^{n} \frac{1}{k}\left|x_{k}-L\right|^{q}+\frac{1}{l_{n}} \sum_{\substack{k=1 \\ k \in K_{\epsilon}}}^{n} \frac{1}{k}\left|x_{k}-L\right|^{q}=S_{1}(n)+S_{2}(n),
$$

where

$$
S_{1}(n)=\frac{1}{l_{n}} \sum_{\substack{k=1 \\ k \notin K_{\epsilon}}}^{n} \frac{1}{k}\left|x_{k}-L\right|^{q} \quad \text { and } \quad S_{2}(n)=\frac{1}{l_{n}} \sum_{\substack{k=1 \\ k \in K_{\epsilon}}}^{n} \frac{1}{k}\left|x_{k}-L\right|^{q} .
$$

Now if $k \notin K_{\epsilon}$ then $S_{1}(n)<\epsilon^{q}$. For $k \in K_{\epsilon}$, we have

$$
S_{2}(n) \leq\left(\sup \left|x_{k}-L\right|\right)\left(\left|K_{\epsilon}\right| / l_{n}\right) \leq M\left|K_{\epsilon}\right| / l_{n} \rightarrow 0
$$

as $n \rightarrow \infty$, since $\delta_{\ln }\left(K_{\epsilon}\right)=0$. Hence $x_{k} \rightarrow L[H, 1]_{q}$.

This completes the proof of the theorem. 
Remark 3.2 The above theorem is analogous to Theorem 2.2 of [15] but with less restrictions on the sequence $x=\left(x_{k}\right)$.

In the next result we characterize statistical summability $(H, 1)$ through the $(H, 1)$ summable subsequences.

Theorem 3.3 A sequence $x=\left(x_{k}\right)$ is statistically summable $(H, 1)$ to $L$ if and only if there exists a set $K=\left\{k_{1}<k_{2}<\cdots<k_{n}<\cdots\right\} \subseteq \mathbb{N}$ such that $\delta(K)=1$ and $(H, 1)-\lim x_{k_{n}}=L$.

Proof Suppose that there exists a set $K=\left\{k_{1}<k_{2}<\cdots<k_{n}<\cdots\right\} \subseteq \mathbb{N}$ such that $\delta(K)=1$ and $(H, 1)-\lim x_{k_{n}}=L$. Then there is a positive integer $N$ such that for $n>N$,

$$
\left|\tau_{n}-L\right|<\epsilon
$$

Put $K_{\epsilon}:=\left\{n \in \mathbb{N}:\left|\tau_{k_{n}}-L\right| \geq \epsilon\right\}$ and $K^{\prime}=\left\{k_{N+1}, k_{N+2}, \ldots\right\}$. Then $\delta\left(K^{\prime}\right)=1$ and $K_{\epsilon} \subseteq \mathbb{N}-K^{\prime}$, which implies that $\delta\left(K_{\epsilon}\right)=0$. Hence $x=\left(x_{k}\right)$ is statistically summable $(H, 1)$ to $L$.

Conversely, let $x=\left(x_{k}\right)$ be statistically summable $(H, 1)$ to $L$. For $r=1,2,3, \ldots$, put $K_{r}:=$ $\left\{j \in \mathbb{N}:\left|\tau_{k_{j}}-L\right| \geq 1 / r\right\}$ and $M_{r}:=\left\{j \in \mathbb{N}:\left|\tau_{k_{j}}-L\right|<1 / r\right\}$. Then $\delta\left(K_{r}\right)=0$ and

$$
M_{1} \supset M_{2} \supset \cdots \supset M_{i} \supset M_{i+1} \supset \cdots
$$

and

$$
\delta\left(M_{r}\right)=1, \quad r=1,2,3, \ldots
$$

Now we have to show that for $j \in M_{r},\left(x_{k_{j}}\right)$ is $(H, 1)$-summable to $L$. Suppose that $\left(x_{k_{j}}\right)$ is not $(H, 1)$-summable to $L$. Therefore there is $\epsilon>0$ such that $\left|\tau_{k_{j}}-L\right| \geq \epsilon$ for infinitely many terms. Let $M_{\epsilon}:=\left\{j \in \mathbb{N}:\left|\tau_{k_{j}}-L\right|<\epsilon\right\}$ and $\epsilon>1 / r(r=1,2,3, \ldots)$. Then

$$
\delta\left(M_{\epsilon}\right)=0,
$$

and by (3.2), $M_{r} \subset M_{\epsilon}$. Hence $\delta\left(M_{r}\right)=0$, which contradicts (3.3) and therefore $\left(x_{k_{j}}\right)$ is $(H, 1)$-convergent to $L$.

This completes the proof of the theorem.

Similarly we can prove the following dual statement.

Theorem 3.4 A sequence $x=\left(x_{k}\right)$ is logarithmic statistically convergent to $L$ if and only if there exists a set $K=\left\{k_{1}<k_{2}<\cdots<k_{n}<\cdots\right\} \subseteq \mathbb{N}$ such that $\delta_{\ln }(K)=1$ and $\lim \frac{x_{k_{n}}}{n}=L$. 


\section{Acknowledgements}

The authors would like to thank the Deanship of Scientific Research at King Abdulaziz University for its financial support under grant number 151-130-1432.

Received: 26 February 2013 Accepted: 12 July 2013 Published: 26 July 2013

\section{References}

1. Fast, H: Sur la convergence statistique. Colloq. Math. 2, 241-244 (1951)

2. Móricz, F: Theorems relating to statistical harmonic summability and ordinary convergence of slowly decreasing or oscillating sequences. Analysis 24, 127-145 (2004)

3. Fridy, JA: On statistical convergence. Analysis 5, 301-313 (1985)

4. Alotaibi, A, Mursaleen, M: A-Statistical summability of Fourier series and Walsh-Fourier series. Appl. Math. Inf. Sci. 6(3), 535-538 (2012)

5. Edely, OHH, Mursaleen, M: On statistical A-summability. Math. Comput. Model. 49, 672-680 (2009)

6. Fridy, JA, Orhan, C: Lacunary statistical convergence. Pac. J. Math. 160, 43-51 (1993)

7. Kolk, E: The statistical convergence in Banach spaces. Tartu Ülik. Toim. 928, 41-52 (1991)

8. Kostyrko, P, Šalát, T, Wilczyński, W: I-Convergence. Real Anal. Exch. 26(2), 669-686 (2000/2001)

9. Mohiuddine, SA, Alotaibi, A, Mursaleen, M: Statistical convergence of double sequences in locally solid Riesz spaces. Abstr. Appl. Anal. 2012, Article ID 719729 (2012). doi:10.1155/2012/719729

10. Mursaleen, M, Alotaibi, A, Mohiuddine, SA: Statistical convergence through de la Vallée-Poussin mean in locally solid Riesz spaces. Adv. Differ. Equ. 2013, 66 (2013). doi:10.1186/1687-1847-2013-66

11. Mursaleen, M, Alotaibi, A: Statistical summability and approximation by de la Vallée-Poussin mean. Appl. Math. Lett. 24, 320-324 (2011) (Erratum: Appl. Math. Lett. 25, 665 (2012))

12. Mursaleen, M, Alotaibi, A: On I-convergence in random 2-normed spaces. Math. Slovaca 61(6), $933-940$ (2011)

13. Mursaleen, M, Edely, OHH: Generalized statistical convergence. Inf. Sci. 162, 287-294 (2004)

14. Mursaleen, M, Edely, OHH: On the invariant mean and statistical convergence. Appl. Math. Lett. 22, 1700-1704 (2009)

15. Mursaleen, M, Karakaya, V, Ertürk, M, Gürsoy, F: Weighted statistical convergence and its application to Korovkin type approximation theorem. Appl. Math. Comput. 218, 9132-9137 (2012)

16. Mursaleen, M, Mohiuddine, SA: On ideal convergence in probabilistic normed spaces. Math. Slovaca 62, 49-62 (2012)

17. Connor, JS: The statistical and strong $p$-Cesàro convergence of sequences. Analysis 8, 47-63 (1988)

18. Ostmann, HH: Additive Zahlentheorie, vol. I. Springer, Berlin (1956)

doi:10.1186/1687-1847-2013-227

Cite this article as: Alghamdi et al.: Logarithmic density and logarithmic statistical convergence. Advances in

Difference Equations 2013 2013:227.

\section{Submit your manuscript to a SpringerOpen ${ }^{\ominus}$ journal and benefit from:}

- Convenient online submission

- Rigorous peer review

- Immediate publication on acceptance

- Open access: articles freely available online

- High visibility within the field

- Retaining the copyright to your article 